The Astrophysical Journal, 676:390-401, 2008 March 20

(C) 2008. The American Astronomical Society. All rights reserved. Printed in U.S.A.

\title{
A SEARCH FOR TEMPERATURE AND DENSITY VARIATIONS IN NGC 7027 WITH THE HUBBLE SPACE TELESCOPE ${ }^{1}$
}

\author{
John H. Bieging and Paul A. Boley \\ Steward Observatory, The University of Arizona, Tucson, AZ 85721; jbieging@as.arizona.edu, pboley@gmail.com \\ William B. LatTer \\ NASA Herschel Science Center, IPAC, California Institute of Technology, Pasadena CA 91125; latter@ipac.caltech.edu
}

AND

A. G. G. M. TIELENS

Kapteyn Astronomical Institute, Postbus 800, 8700 AV Groningen, Netherlands; tielens@astro.rug.nl Received 2007 June 14; accepted 2007 December 13

\begin{abstract}
We observed the young planetary nebula NGC 7027 with the HST Imaging Spectrograph (STIS) in long-slit mode at five slit positions along the major axis of the nebula, over the wavelength range from 3000 to $\sim 10,000 \AA$. We used dereddened line ratios to determine line-of-sight average values for (1) $\mathrm{He}^{++}$and $\mathrm{He}^{+}$ionization fractions from He II $\lambda 4686$ and He I 25876 ; (2) electron density from both [S II] ( $\lambda 6716 / \lambda 6731)$ and [Ar IV] ( $\lambda 4711 / \lambda 4740)$; and (3) temperature from $\left[\mathrm{O}\right.$ III] $(\lambda 4959+\lambda 5007) / \lambda 4363$. The $\mathrm{He}^{++} / \mathrm{H}^{+}$ratio varies across the nebula, with values up to 0.06 over the central parts, falling off to 0.03 at offsets of $5^{\prime \prime}-6^{\prime \prime}$ from the central star. The line-of-sight mean densities vary by factors of $\sim 5$ on angular scales as small as $\sim 1^{\prime \prime}$. The average electron temperatures are generally consistent with a constant $\left\langle T_{e}\right\rangle=13,800 \pm 2000 \mathrm{~K}$ within the uncertainties, except for $\sim 2 \%$ of measured pixels where $T_{e}>$ $18,000 \mathrm{~K}$. The variations of density along the slits are not obviously correlated with variations in extinction. The ionization structure of the nebula varies on both large and small scales. [O I] $\lambda 6300, \mathrm{H} \alpha$, and $\mathrm{He}$ II $\lambda 4686$ intensity profiles along each slit appear to trace ionization gradients at $\mathrm{H} \alpha$ peaks, with the highest ionization on the side toward the central star and lowest ionization on the far side. These structures may result from effects of stellar radiation, shocks from a fast stellar wind, or some combination of the two processes.
\end{abstract}

Subject headings: atomic processes — planetary nebulae: general — planetary nebulae: individual (NGC 7027)

\section{INTRODUCTION}

The complex structure of planetary nebulae $(\mathrm{PNe})$ was revealed with striking clarity by the Hubble Space Telescope (HST), both in optical images of the ionized gas (e.g., Sahai \& Trauger 1998; Ciardullo et al. 1999; Sahai 2003) and in near-infrared images of the ionized-neutral interface or photodissociation region (PDR; e.g., Latter et al. 2000) in young PNe. Despite this wealth of evidence for small-scale structure in the density of the ionized gas, there is a continuing debate over the temperature structure in the gas. This debate is motivated largely by the long-standing discrepancy between the ionic abundances derived from collisionally excited forbidden lines on the one hand, and ionic recombination lines on the other (see reviews by Mathis 1995; Peimbert 1995; Liu 2003, 2006; Torres-Peimbert \& Peimbert 2003; Peimbert \& Peimbert 2006). Peimbert (1967) first proposed that small-scale spatial variations in temperature integrated along sight lines or over whole nebulae would yield discrepant abundances as a result of the very different temperature dependences of the volume emissivities of forbidden lines compared with recombination lines. An alternative explanation has been proposed more recently (see Liu 2003) where PNe are postulated to contain small-scale inclusions comprising a small fraction of the nebular mass, which are enhanced in metals or deficient in hydrogen. Strong cooling in these inclusions results in greatly decreased temperatures $\left(T_{e} \sim 10^{3} \mathrm{~K}\right)$ with consequently enhanced recombination line emission but de-

\footnotetext{
${ }^{1}$ Based on observations with the NASA/ESA Hubble Space Telescope obtained at the Space Telescope Science Institute, which is operated by the Association of Universities for Research in Astronomy, Incorporated, under NASA contract NAS5-26555.
}

creased forbidden line emission relative to the bulk of the nebula. Detailed models with such inclusions (e.g., Liu et al. 2000; Pequignot et al. 2003) have been presented to account for several PNe with highly discrepant abundance determinations.

Several studies have attempted to measure spatial variations of temperature in PNe. Observational techniques used include imaging through narrowband filters centered at the relevant optical emission lines (e.g., [O III] $\lambda \lambda 4363$ and $5007 \AA$ from Rubin et al. [2002] and Wesson \& Liu [2004] and [N II] from Lame \& Pogge [1994]) and long-slit spectroscopy (e.g., Liu et al. 2000; Garnett $\&$ Dinerstein 2001; Rubin et al. 2002; Monteiro et al. 2004). Both techniques have been applied to well-resolved nebulae in order to achieve good spatial discrimination of temperature differences. These studies have not shown large temperature variations either on small spatial scales or systematically over the nebulae, in any of the objects studied to date. A fundamental limitation to such studies is, of course, that the observed light is integrated along the line of sight through the nebula, so that any temperature or density structure in that direction may be masked by the radiative transfer. Small-scale temperature variations, as proposed by Peimbert (1967), could therefore be present but washed out in the observed spectra. This effect would be most severe for low spatial resolution ground-based spectra taken in moderate seeing. With the high angular resolution achievable in space with $H S T$, spatial variations in nebular temperature and density might be more readily discernible provided that the spatial scale and filling factor of the variations had suitable values.

The subject of the present study, NGC 7027, is a very young PN with a dynamical age of $\sim 600$ yr obtained by Masson (1989) for his derived distance of 900 pc (although Bains et al. [2003] 
find a lower distance, $650 \mathrm{pc}$, based on a revised kinematic model). The central star has a Zanstra temperature of $~ 200,000 \mathrm{~K}$ (Jacoby 1988; Robberto et al. 1993; Latter et al. 2000) and inferred progenitor mass of $4 M_{\odot}$, so it is observed at close to the maximum temperature on theoretical evolutionary tracks for PNe central stars with such initial masses (Blöcker 1995). The PN was discovered to be an X-ray source by Kastner et al. (2001), who attributed the $\mathrm{X}$-ray emission to shock heating by a fast wind from the central star impacting the slow wind which the progenitor star ejected while on the asymptotic giant branch (AGB). The existence of a slow progenitor AGB wind is unequivocal from observations of a massive $\left(\sim 3 M_{\odot}\right)$, roughly spherical expanding envelope mapped in emission lines of CO and other molecules (Bieging et al. 1991; Jaminet et al. 1991), as well as infrared images of vibrationally excited $\mathrm{H}_{2}$ from a PDR between the neutral molecular envelope and the ionized zone of the PN (Latter et al. 2000; Graham et al. 1993). The nebula also shows a bipolar jetlike feature in the near-IR (Latter et al. 2000; Cox et al. 2002), which may be a further manifestation of a fast collimated outflow from the central star (or from an undetected binary companion).

Seen in its entirety then, NGC 7027 exhibits an extraordinary range of physical conditions and phenomena, centered on an extremely hot central star and its surrounding photoionized nebula ( $T_{e} \approx 10^{4} \mathrm{~K}$, but with some component of X-ray emitting gas at temperatures of $\sim 8 \times 10^{6} \mathrm{~K}$; Maness et al. 2003). This ionized zone is embedded in a massive neutral envelope on which the hot central star may be exerting a dynamical force via a fast stellar wind, in addition to the effect of photoionization. Within this complex structure, one might well expect to find evidence for a nonuniform temperature distribution in the ionized gas. In this paper we present observations of NGC 7027 made with the HST Imaging Spectrograph (STIS). We have applied standard nebular density and temperature diagnostics from optical emission lines to examine the spatial structure of the ionized gas, taking advantage of the high angular resolution $\left(\sim 0.1^{\prime \prime}\right)$ afforded by the long-slit spectroscopic mode of STIS.

\section{OBSERVATIONS AND DATA ANALYSIS}

NGC 7027 was observed on 2000 January 22 with STIS in its highest spatial resolution mode, with the $52^{\prime \prime} \times 0.1^{\prime \prime}$ slit. Exposures were taken with the slit aligned approximately along the major axis of the nebula at five positions equispaced to sample the nebula from the edge of the ionized zone/PDR interface (slit position 1) to the brightest parts of the visible nebula (position 5). The locations of the slits are shown in Figure 1, overlaid on an HST optical image from the program of Bond et al. (HST proposal 6119; see Ciardullo et al. 1999). Throughout this paper, coordinates for positions along each slit are referenced to the fiducial marks shown in Figure 1.

Each slit position was observed with three gratings selected to cover the entire visible spectrum in two segments (2850-5750 $\AA$ and 5250-10,200 $\AA$ ) at low resolution, and one segment (6295$6865 \AA$ ) at medium resolution $(1.1 \AA$ ). The grating settings, resolutions, and exposure times per slit are summarized in Table 1. All calibrations were done using standard procedures for flatfielding (from contemporaneous flats) and calibration of wavelength and intensity. The data products from the reduction pipeline were delivered by the Space Telescope Science Institute for further analysis. The two-dimensional spectra as delivered had been rectified so that the dispersion and spatial axes were orthogonal, with the wavelength scale referred to vacuum. The spatial dimension had pixels of $0.05^{\prime \prime}$ width, thus providing Nyquist sampling of the nominal HST point-spread function. The intensity scale was calibrated in standard cgs units. The flux calibration takes into account the variation of the point-spread function with wavelength along the dispersion axis. The accuracy of the rectification is 0.2 pixels in the wavelength axis, and between 0.2 and 0.5 pixels in the spatial axis, with the lower value typical of the central portion ( $20 \%$ ) of the slit which NGC 7027 covers. Since the data were binned along the spatial axis by 11 pixels (or 21 pixels in some cases) and the spectral line apertures were typically about 8 pixels ( 4 resolution elements) wide, the rectification accuracy should not be a significant source of uncertainty in deriving line ratios.

Examples of the resultant spectra are in Figure 2, which shows spectra for the three gratings integrated over $\pm 8^{\prime \prime}$ along slit 4 in Figure 1. In the expanded-scale plots (lower half of each panel), some sharp positive or negative features are visible that are effects of cosmic rays not removed by the data processing pipeline. These artifacts were removed from the spatial intensity profiles of individual spectral lines by the method described below.

Spatial profiles of spectral lines for the five slit positions were made by integrating the intensity along the dispersion axis at each pixel along the position axis using the IRAF task sbands. Spectral apertures for the integration along the dispersion axis were selected by plotting the spatially integrated intensity as a function of wavelength and choosing limits that included the entire width of the spectral line, but as little continuum as possible. Apertures for lines of interest are given in Table 2. Apertures for determining the continuum were selected from adjacent line-free regions of the spectrum, with bandwidths taken as large as possible to reduce noise but avoid contamination by other spectral lines. Absence of strong contaminating line emission is confirmed by the deep spatially integrated spectrum of Zhang et al. (2005). The resulting intensity profiles as a function of spatial location along the slit were then used in all subsequent calculations.

Prior to combining any spectral line intensity profiles, boxcar smoothing in the spatial coordinate was applied to reduce random variations due to noise. We typically used a width of 11 pixels, corresponding to a spatial smoothing of $0.55^{\prime \prime}$.

Although the observations were made in the "accumulate" mode and cosmic-ray rejection was applied to the target exposures, we found that the data were still significantly contaminated by cosmic rays (CRs). The CRs exhibited several properties which aided in their identification and removal. For a given spectral line, CRs almost always occurred at the same spatial pixel across all five slits, a characteristic that implies these are artifacts in the calibration exposures which were applied in common to all of the five exposures at the different slit positions. The CR features are always very sharp, typically confined to one or two pixels. The algorithm used for CR identification exploited these traits. Pixels with large spatial first derivatives (several times the rms noise) in several slits (typically three or more) were flagged as CRs. Once a CR had been identified by the algorithm, it was corrected for by linearly interpolating a new intensity value from neighboring, unaffected pixels in the intensity spatial profile.

Dereddening was done on a per-pixel basis using a standard extinction law. The observed intensity $I(\lambda)$ at a wavelength $\lambda$ is related to the unextincted intensity $I_{0}(\lambda)$ by

$$
\frac{I(\lambda)}{I(\mathrm{H} \beta)}=\frac{I_{0}(\lambda)}{I_{0}(\mathrm{H} \beta)} 10^{-c[f(\lambda)-f(\mathrm{H} \beta)]},
$$

where we used the standard $(R=3.1)$ reddening curve $f(\lambda)$ from Table 7.1 in Osterbrock \& Ferland (2006). The dereddening parameter $c$ was determined from the observed $\mathrm{H} \alpha / \mathrm{H} \beta$ intensity ratio and varies substantially with position across the nebula. The intrinsic ratio of the $\mathrm{H} \alpha / \mathrm{H} \beta$ intensities varies with temperature, 


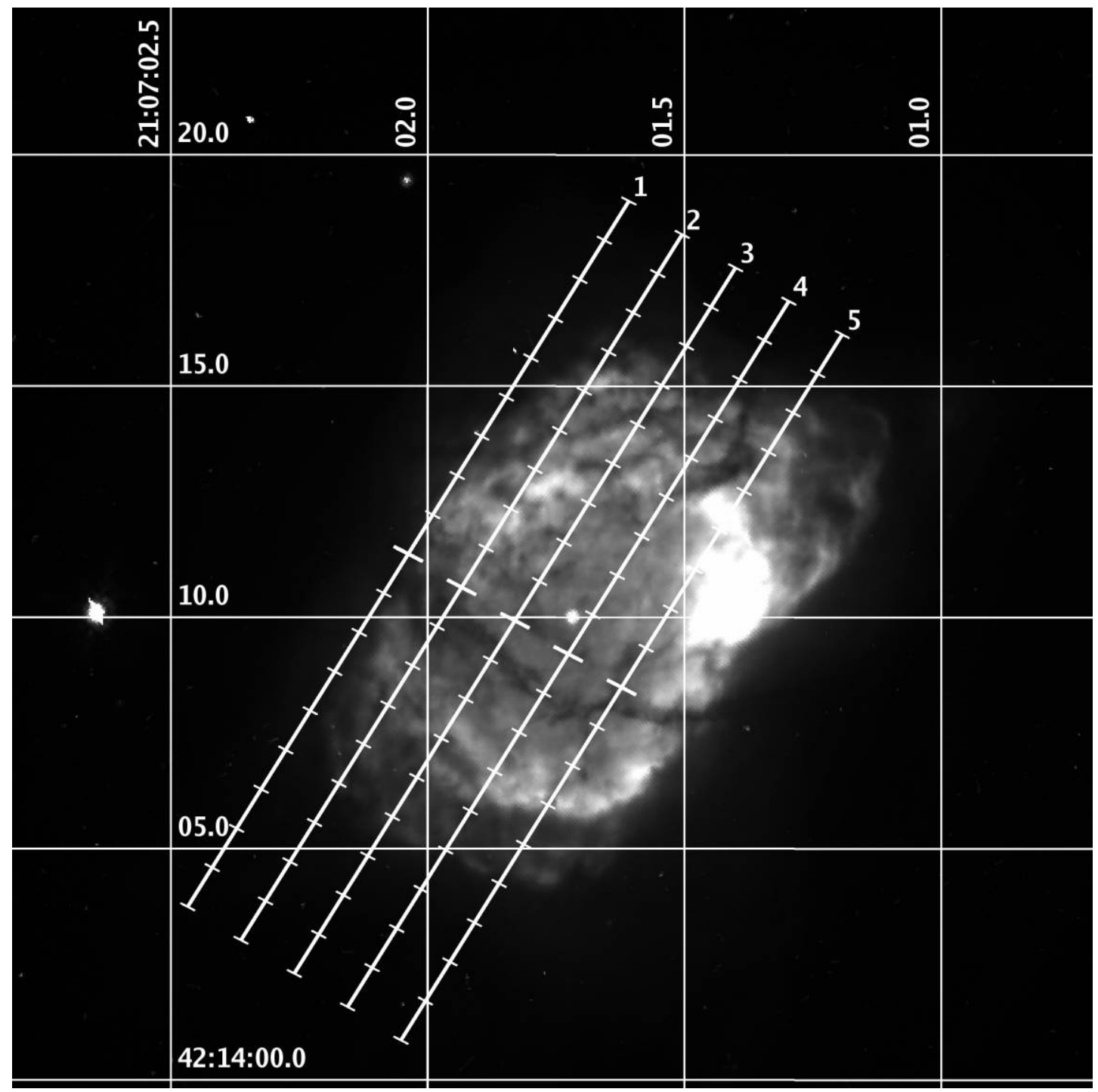

FIG. 1. - HST WFPC2 F555W image of NGC 7027 from program of Bond et al. (HST proposal 6119; see Ciardullo et al. 1999) with the five slit positions overlaid. Positions along each slit are measured relative to the longer tick mark, positive offsets toward the top. Interval between ticks is $1^{\prime \prime}$. The slit width was $0.1^{\prime \prime}$, which is approximately the thickness of the lines showing the slit locations.

but was taken to be a constant value of 2.80 , calculated for a temperature of $13,000 \mathrm{~K}$ and $n_{e}$ of $60,000 \mathrm{~cm}^{-3}$ by interpolation in Table 4.4 in Osterbrock \& Ferland (2006). These values for temperature and density are consistent with those derived by Zhang et al. (2005) from their sensitive, high-resolution nebular-averaged spectrum. The uncertainty in $c$ was calculated using standard error propagation formulae. The standard deviation for each spectral line profile was determined by computing the rms for a few hundred pixels located off the nebular emission. Spatial and spectral smoothing reduce the photon counting noise sufficiently to be omitted in the uncertainty calculation.

TABLE 1

Gratings and Exposures at Each Slit Position

\begin{tabular}{ccccc}
\hline \hline Grating & $\begin{array}{c}\text { Central Wavelength } \\
(\AA)\end{array}$ & $\begin{array}{c}\text { Spectral Range } \\
(\AA)\end{array}$ & $\begin{array}{c}\text { Resolution } \\
(\AA)\end{array}$ & $\begin{array}{c}\text { Integ. Time } \\
(\mathrm{s})\end{array}$ \\
\hline G430L ...... & 4300 & 2900 & 5.5 & 115 \\
G750L ...... & 7751 & 5030 & 9.8 & 115 \\
G750M ..... & 6581 & 570 & 1.1 & 270 \\
\hline
\end{tabular}

The reddening correction factor $c$ together with $1 \sigma$ error bars is shown for each of the five slit locations in Figure 3. Values of $c$ vary significantly across the nebula. The largest reddening occurs in slits 1 and 2, which lie closest to the edge of the visible ionized region. Our dereddening prescription assumes, essentially, a foreground screen model with spatial variations. NGC 7027 shows much structure in its optical appearance (Ciardullo et al. 1999), which may indicate that some of the reddening is internal to the nebula. The trend in Figure 3 of increased reddening toward the edge of the nebula (i.e., slit positions 1 and 2) is consistent with significant internal reddening. If so, the assumption of a standard interstellar reddening law may be incorrect since this object is very carbon-rich and its associated dust may have different extinction properties than silicate-rich interstellar grains. Seaton (1979) found, however, that the standard interstellar extinction law gives an excellent fit to the nebular-averaged reddening from $\mathrm{H} \alpha$ to $1640 \AA$, and Osterbrock (1974) estimated that no more than 0.6 mag of extinction at $\mathrm{H} \beta$ is produced by internal dust, out of a total of $3.6 \mathrm{mag}$. Atherton et al. (1979) derived a relative extinction map which resembled the symmetry of the $5 \mathrm{GHz}$ radio continuum. They argued, therefore, that the bulk of the absorbing 

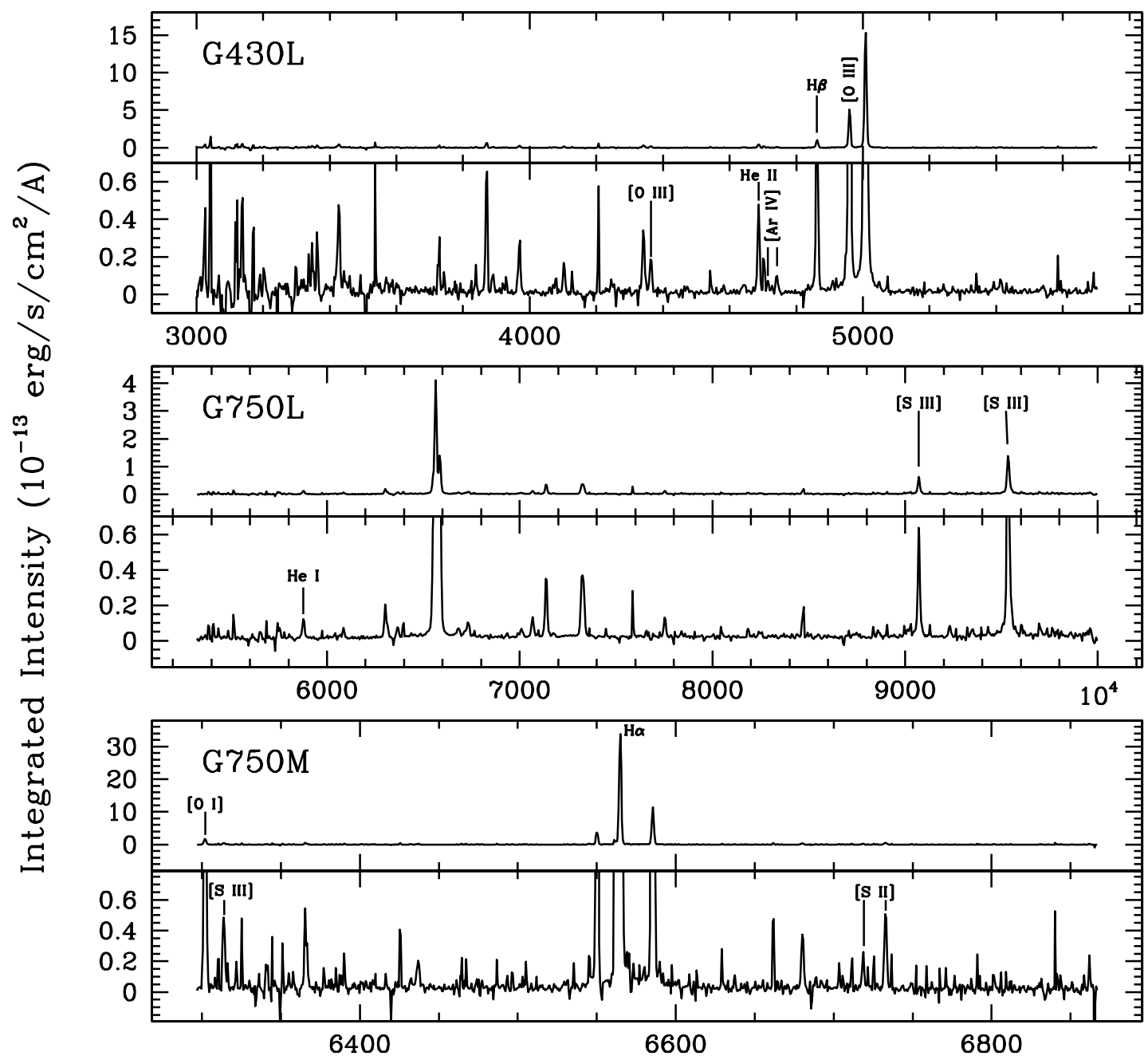

Wavelength (Angstroms)

FIG. 2.-Examples of observed spectra for the three gratings, integrated over $\pm 8^{\prime \prime}$ along slit 4 . The lower half of each panel has an expanded vertical scale; some of the visible sharp spikes are residual cosmic-ray artifacts, which were removed in subsequent processing (see text). Spectral lines from Table 2 are marked.

TABLE 2

Spectral Line Extraction Apertures

\begin{tabular}{|c|c|c|c|}
\hline Line ID & Grating & $\begin{array}{c}\text { Center } \\
(\AA \AA)\end{array}$ & $\begin{array}{c}\text { Width } \\
(\AA)\end{array}$ \\
\hline$[\mathrm{O}$ III] $\lambda 4363 \ldots \ldots \ldots \ldots \ldots \ldots \ldots \ldots$ & G430L & 4364 & 20 \\
\hline Не ІІ $\lambda 4686 \ldots \ldots \ldots \ldots \ldots \ldots \ldots \ldots$. & G430L & 4687 & 20 \\
\hline$[$ Ar IV] $\lambda 4711 \ldots \ldots \ldots \ldots \ldots \ldots$ & G430L & 4712.5 & 20 \\
\hline [Ar IV] $\lambda 4740 \ldots \ldots \ldots \ldots \ldots \ldots \ldots$ & G430L & 4742 & 20 \\
\hline $\mathrm{H} \beta$ & G430L & 4862 & 20 \\
\hline 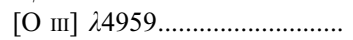 & G430L & 4960 & 30 \\
\hline$[\mathrm{O}$ ІІІ] $25007 \ldots \ldots \ldots \ldots \ldots \ldots \ldots$ & G430L & 5009 & 30 \\
\hline Не І 25876 & G750L & 5877 & 30 \\
\hline [O I] $\lambda 6300 \ldots \ldots \ldots \ldots \ldots \ldots . . .$. & G750M & 6302 & 4 \\
\hline [S III] $\lambda 6312$ & G750M & 6312 & 4 \\
\hline $\mathrm{H} \alpha$ & G750M & 6565 & 5 \\
\hline [S II] $\lambda 6716 \ldots \ldots \ldots \ldots \ldots \ldots$ & G750M & 6719 & 4 \\
\hline [S II] $\lambda 6731 \ldots \ldots \ldots \ldots \ldots \ldots$ & G750M & 6733 & 4 \\
\hline 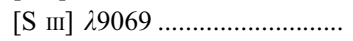 & G750L & 9070 & 30 \\
\hline 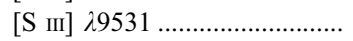 & G750L & 9532 & 30 \\
\hline
\end{tabular}

material was close but external to the ionized gas. Woodward et al. (1992) compared $\mathrm{H} \alpha, 5 \mathrm{GHz}$ radio, and IR images to derive a relative $(H-K)$ color excess map. The morphology of their map agreed with that of Atherton et al. (1979), showing a U-shaped maximum of extinction with a minimum in a trough along the symmetry axis of, and centered on, the radio continuum. Woodward et al. (1992) suggested that the obscuring dust lies in a shell or disk exterior to the ionized gas, possibly a part of the neutral molecular envelope that surrounds the nebula.

Walton et al. (1988) derived a quantitative extinction map of $E(B-V)$ by comparing $\mathrm{H} \beta$ and $15 \mathrm{GHz}$ radio continuum images. They also assumed a foreground screen model, concluding that the dust is predominantly associated with the nebula, but has a higher density in the outer parts, although some extinction is probably internal to the ionized nebula. We have extracted linear cuts from the $E(B-V)$ contour map of Walton et al. (1988), corresponding to slits $2-5$ (slit 1 lies at the edge of their map), and converted to $c(\mathrm{H} \beta)$ using their factor of 1.46 . Figure 4 compares our values of $c(\mathrm{H} \beta)$ with those from Walton et al. (1988). Note that the spatial sampling differs between these data sets. The extinction map of Walton et al. (1988) has a 1.3" (FWHM) circular Gaussian PSF, while our $c(\mathrm{H} \beta)$ displayed in Figure 4 is boxcar smoothed by 21 pixels, or $1.05^{\prime \prime}$ along the $0.1^{\prime \prime}$ wide slits. The 


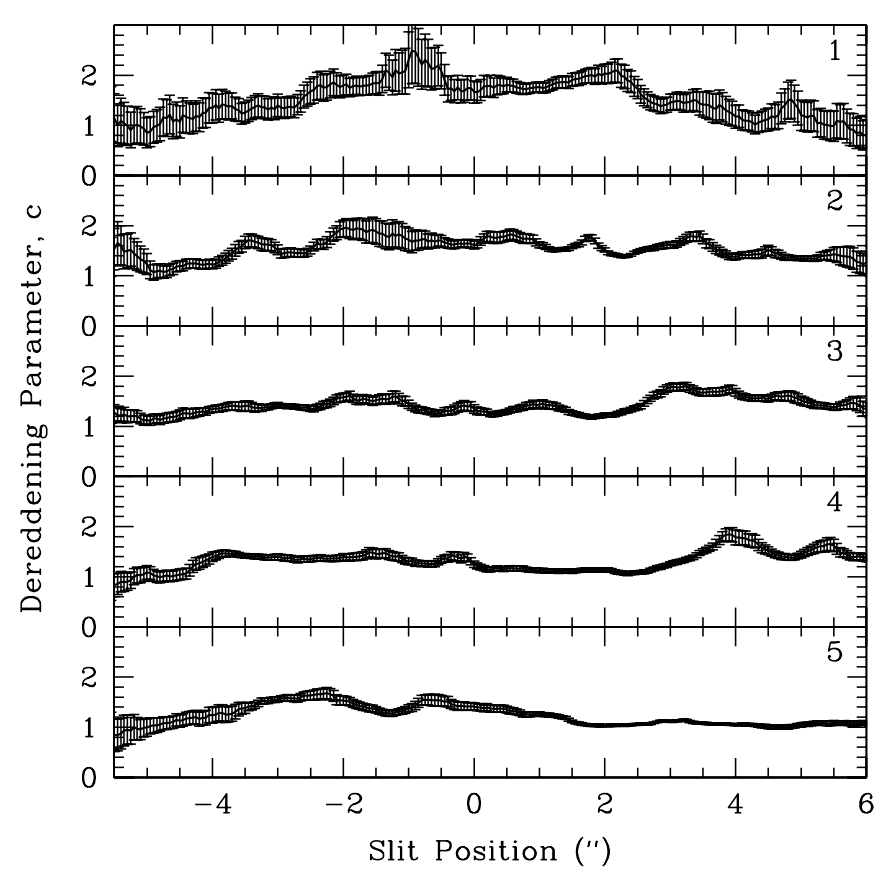

FIG. 3.- Reddening correction, $c(\mathrm{H} \beta)$, as a function of slit position for the five slit locations shown in Fig. 1. The $\mathrm{H} \alpha$ and $\mathrm{H} \beta$ intensity profiles have been boxcar smoothed by 11 pixels $\left(0.55^{\prime \prime}\right)$ along each slit before computing $c$ from the standard reddening curve (see text).

shapes of the two curves are similar for each slit, with the best agreement in slits 4 and 5, where the gradient in the Walton et al. $E(B-V)$ map perpendicular to the slits is small. For slit 2, $E(B-V)$ varies significantly within $\sim 1^{\prime \prime}$ perpendicular to the slit, which probably explains the discrepancy in absolute level of

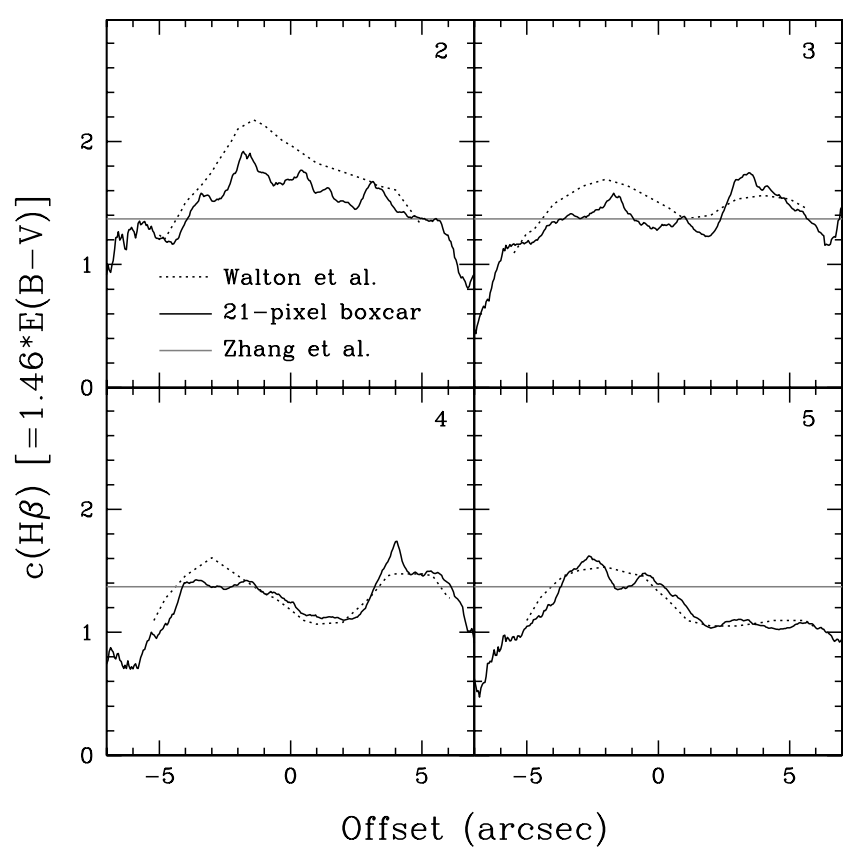

FIG. 4.-Comparison of our reddening correction, $c(\mathrm{H} \beta)$, as a function of slit position for slits $2-5$ (see Fig. 1), with cuts through the $E(B-V)$ map by Walton et al. (1988). The $\mathrm{H} \alpha$ and $\mathrm{H} \beta$ intensity profiles were boxcar smoothed by 21 pixels $\left(1.05^{\prime \prime}\right)$ along each slit before computing $c$ from the standard reddening curve (see text), so the effective aperture for which the solid curves are derived is $0.1^{\prime \prime} \times$ $1.05^{\prime \prime}$, while the resolution of the Walton et al. extinction map is a circular Gaussian with FWHM $\sim 1.3^{\prime \prime}$. The thin solid horizontal line shows the nebular average value of 1.37 derived by Zhang et al. (2005).

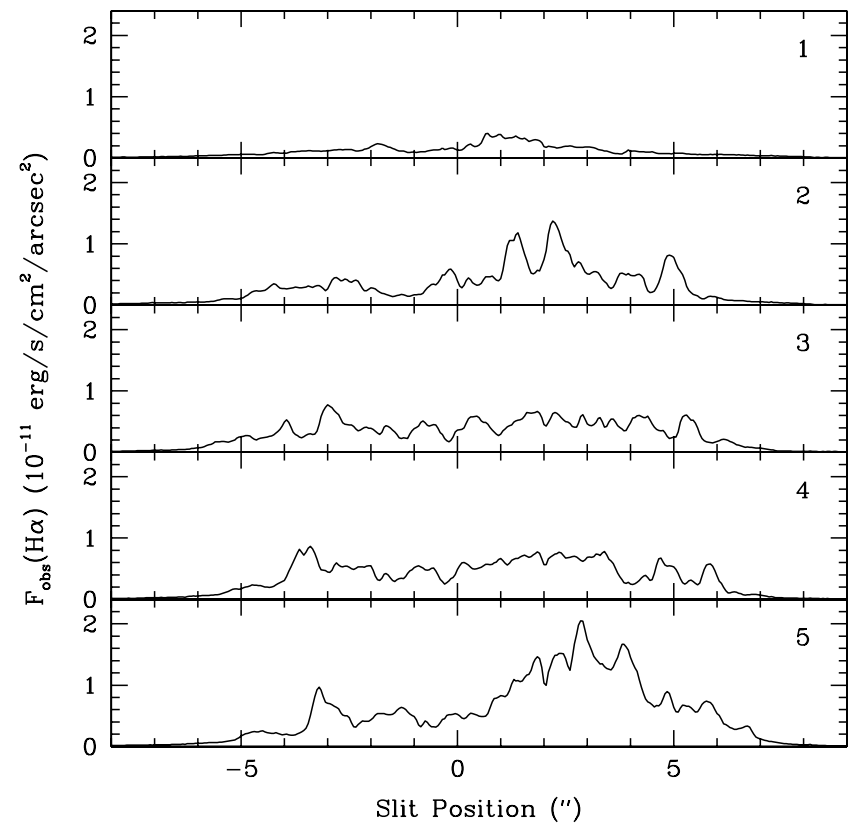

FIG. 5.-Observed H $\alpha$ flux as a function of slit position for the five slit locations shown in Fig. 1. These data have not been smoothed.

$c(\mathrm{H} \beta)$. Given the differences in spatial sampling, there is good agreement in the magnitude and spatial variation of our derived reddening parameter and that of Walton et al. (1988).

To derive the temperature and density as a function of position along each slit, we developed a custom software package which applied the smoothing and dereddening, summed lines together as necessary, subtracted continua or other contaminant lines, and formed the relevant line intensity ratios for temperature and density diagnostics. This software propagates all uncertainties due to rms noise, dereddening, smoothing, and continuum subtraction, according to standard error propagation formulae. The final output is a file containing the line ratio and its uncertainty across the five spatial profiles.

Temperatures and densities were determined using the IRAF task temden for standard diagnostic line ratios (Shaw \& Dufour 1995). Tables of temden output were created, giving the temperature as a function of intensity ratio and density, and density as a function of line ratio and temperature. Temperatures and densities as a function of slit position were then calculated by bilinear interpolation from these tables.

\section{RESULTS}

\section{1. $\mathrm{H} \alpha$ and He Recombination Lines}

Spatial profiles of the observed $\mathrm{H} \alpha$ flux, $F_{\text {obs }}(\mathrm{H} \alpha)$, unsmoothed and uncorrected for extinction, along each of the five slit positions are shown in Figure 5. The relatively weak emission in slit 1 results from the location tangent to the ionized zone/PDR interface and correspondingly high extinction. Consequently, many of the standard nebular diagnostic lines are too weak to give accurate temperatures or densities for slit 1 . Slits 2, 3, and 4 all have comparable mean observed $\mathrm{H} \alpha$ fluxes but there are significant variations over small angular scales in these profiles, by factors up to $2-3$ within $0.3^{\prime \prime}$ along the slit, notably for slit 2 . Slit 5 crosses one of the brightest parts of the optical nebula with correspondingly high values of $F_{\text {obs }}(\mathrm{H} \alpha)$. The variation in $F_{\text {obs }}(\mathrm{H} \alpha)$ may result from structure in the foreground dust produced by stellar mass loss when the central star was on the AGB, and possibly from small-scale structure in the density of the ionized gas itself. 


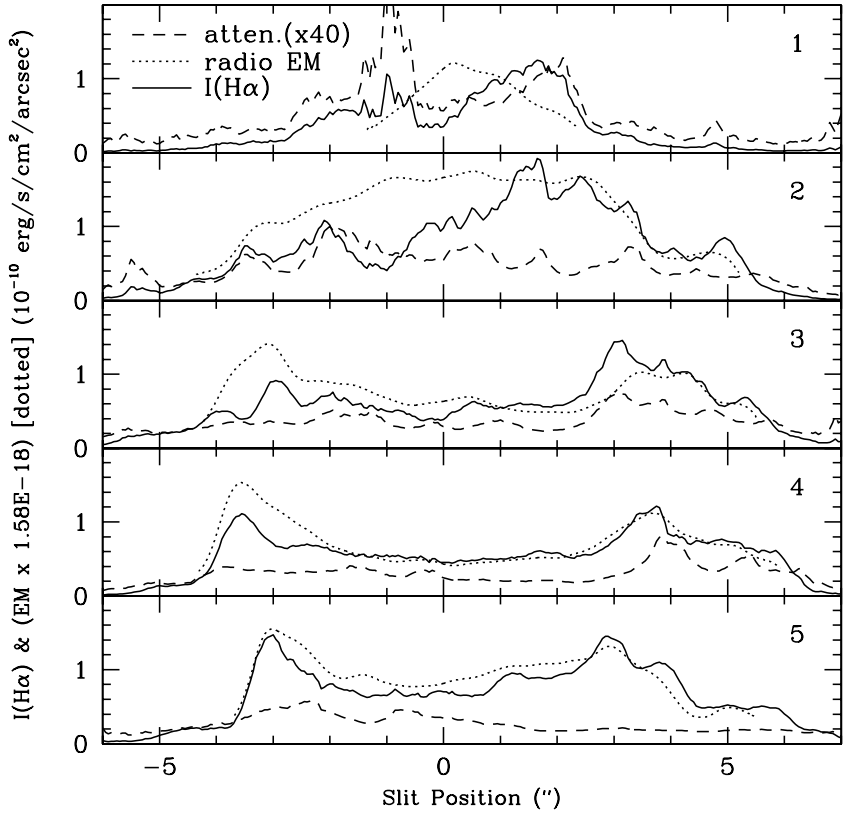

FIG. 6- - Solid line: Extinction-corrected $\mathrm{H} \alpha$ intensity after spatial smoothing by 11 pixels $\left(0.55^{\prime \prime}\right)$; dashed line: extinction attenuation factor at $\mathrm{H} \alpha$; dotted line: radio-derived emission measure scaled by a factor of $1.58 \times 10^{-18}$, which is the predicted $\mathrm{H} \alpha$ intensity for $T_{e}=13,000 \mathrm{~K}$. Emission measures are taken from cuts along the five slit positions extracted from the map of Bains et al. (2003).

Before applying extinction corrections it was necessary to smooth the emission line profiles in the spatial coordinate to avoid increasing the noise level excessively, mainly due to the large extinction at $\mathrm{H} \beta$ which is used to derive $c$. The $\mathrm{H} \alpha$ intensity corrected for extinction, $I(\mathrm{H} \alpha)$, is shown in Figure 6. The $\mathrm{H} \alpha$ data have been boxcar smoothed by 11 pixels $\left(0.55^{\prime \prime}\right)$ and corrected by the attenuation factor, $10^{c f(\lambda)}$, which is plotted in Figure 6 as a dashed line. The dereddened $I(\mathrm{H} \alpha)$ profiles are more symmetric about the center of the nebula than the observed profiles, consistent with the morphology of the ionized gas at radio and IR wavelengths.

We can also compare the $I(\mathrm{H} \alpha)$ spatial profiles directly with the emission measure derived by Bains et al. (2003) from highresolution radio continuum maps, which are unaffected by dust extinction. Assuming $T_{e}=13,000 \mathrm{~K}$ and $n_{e}=60,000 \mathrm{~cm}^{-3}$, the surface brightness of $\mathrm{H} \alpha$ should scale with emission measure, EM, as

$$
I(\mathrm{H} \alpha)=1.58 \times 10^{-18} \mathrm{EM} \mathrm{erg} \mathrm{s}{ }^{-1} \mathrm{~cm}^{-2} \operatorname{arcsec}^{-2},
$$

where EM is in $\mathrm{cm}^{-6} \mathrm{pc}$ and the coefficient was derived by interpolation from the emissivity tables of Storey \& Hummer (1995). The resulting spatial profiles for linear cuts through the Bains et al. (2003) EM map are shown as dotted lines in Figure 6. It is important to note that the radio-derived EM map has a circular Gaussian beam with a FWHM of $0.4^{\prime \prime}$, while the $I(\mathrm{H} \alpha)$ profiles have an effective spatial aperture of $0.1^{\prime \prime} \times 0.55^{\prime \prime}$. The best agreement between $I(\mathrm{H} \alpha)$ and scaled EM in Figure 6 is for regions where the EM map does not vary greatly perpendicular to the slit long axis. There is good agreement for slits 3, 4, and 5, especially in the central $\sim 6^{\prime \prime}$, where the EM gradient is small. For slits 1 and 2, the EM changes rapidly perpendicular to the slit axis, so the radio EM map samples a significantly different range of values than the optical spectra, and the agreement with $I(\mathrm{H} \alpha)$ is relatively poorer. Along slit 4 in the range $-2^{\prime \prime}$ to $+6^{\prime \prime}$, the EM varies only slightly perpendicular to the slit and over this region the agreement between our dereddened $I(\mathrm{H} \alpha)$ and the scaled radio EM is excellent.

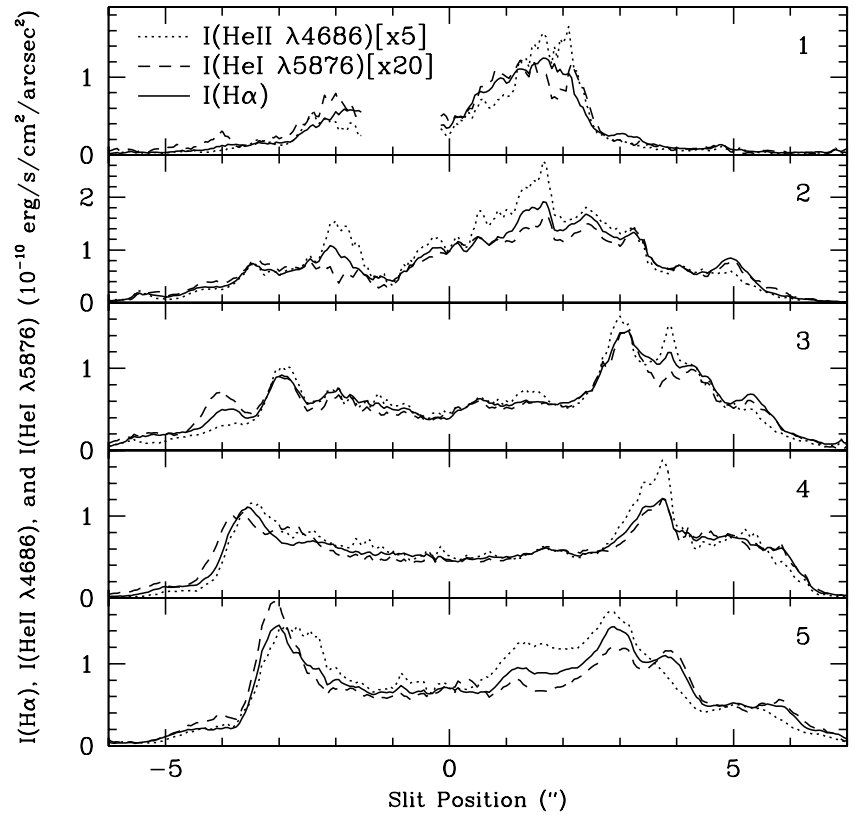

FIG. 7.-Comparison of dereddened He II $\lambda 4686$ and He I $\lambda 5876$ with $\mathrm{H} \alpha$ for the five slit locations shown in Fig. 1. The data were boxcar smoothed over 11 pixels $\left(0.55^{\prime \prime}\right)$ and then dereddened.

The He II $\lambda 4686$ recombination line is prominent in the spectrum of NGC 7027, as expected for a central star with a Zanstra temperature of $200,000 \mathrm{~K}$. The $\lambda 5876$ recombination line of $\mathrm{He} \mathrm{I}$ is also well detected in our spectra. Figure 7 shows the dereddened spatial profiles of $I(\mathrm{He}$ II 24686$)$ and $I(\mathrm{He}$ I 25876$)$, compared with $I(\mathrm{H} \alpha)$ for the five slit positions. These three lines have generally very similar profiles, but there are differences in detail. We have calculated the line-of-sight averaged ionic abundance ratios $\mathrm{He}^{++} / \mathrm{H}^{+}, \mathrm{He}^{+} / \mathrm{H}^{+}$, and $\left(\mathrm{He}^{++}+\mathrm{He}^{+}\right) / \mathrm{H}^{+}$from the profiles in Figure 7, using line emissivities interpolated from Storey \& Hummer (1995) and Benjamin et al. (1999), for $T_{e}=13,000 \mathrm{~K}$. The resulting ratios are shown in Figure 8. (The graphs are blanked where $I(\mathrm{H} \alpha)<2 \times 10^{-11} \mathrm{erg} \mathrm{s}^{-1} \mathrm{~cm}^{-2} \operatorname{arcsec}^{-2}$, where the ionic ratios become unreliable.) From their sensitive nebular-averaged spectrum, Zhang et al. (2005) found $\mathrm{He}^{++} / \mathrm{H}^{+}=0.041, \mathrm{He}^{+} / \mathrm{H}^{+}=$ 0.058 , and deduced a total elemental abundance of $\mathrm{He} / \mathrm{H}=0.10$ on the assumption that both $\mathrm{He}$ and $\mathrm{H}$ are ionized throughout the nebula. The thin horizontal lines in the panels of Figure 8 mark this average total $\mathrm{He} / \mathrm{H}$ abundance. (Analyses of spectra restricted to the brightest part of the nebula by Bernard Salas et al. [2001] and Keyes et al. [1990] found slightly higher values, 0.107 and 0.112 respectively.) The dotted lines in Figure 8 show the $\mathrm{He}^{++} / \mathrm{H}^{+}$ abundance and the dashed lines show the $\mathrm{He}^{+} / \mathrm{H}^{+}$abundance. The solid lines are the sum of the $\mathrm{He}^{++} / \mathrm{H}^{+}$and $\mathrm{He}^{+} / \mathrm{H}^{+}$abundances, which should give the total elemental abundance, $\mathrm{He} / \mathrm{H}$, if both $\mathrm{He}$ and $\mathrm{H}$ are ionized throughout the nebula. The relative fractions of $\mathrm{He}^{++}$and $\mathrm{He}^{+}$vary somewhat with position, but are generally consistent with the nebular averages of Zhang et al. (2005). There is a slight trend that at positions more than $4^{\prime \prime}$ from the center along the slits, $\mathrm{He}^{++} / \mathrm{H}^{+}$is lower than the mean, and is higher than the mean for positions within $\sim \pm 3^{\prime \prime}$ of the center. The $\mathrm{He}^{+} / \mathrm{H}^{+}$ratio is nearly constant at $\sim 0.06$ over most slit positions, but rises somewhat at slit positions more than $4^{\prime \prime}$ from the center.

The total ionic number density ratio, $\left(\mathrm{He}^{++}+\mathrm{He}^{+}\right) / \mathrm{H}^{+}$, is within $20 \%$ of the nebular average of 0.10 over $85 \%-90 \%$ of the pixels plotted in slits 2-5 in Figure 8. This agreement indicates that the reddening corrections and the flux calibrations for these lines are reliable over at least these portions of the spatial profiles. For slits 


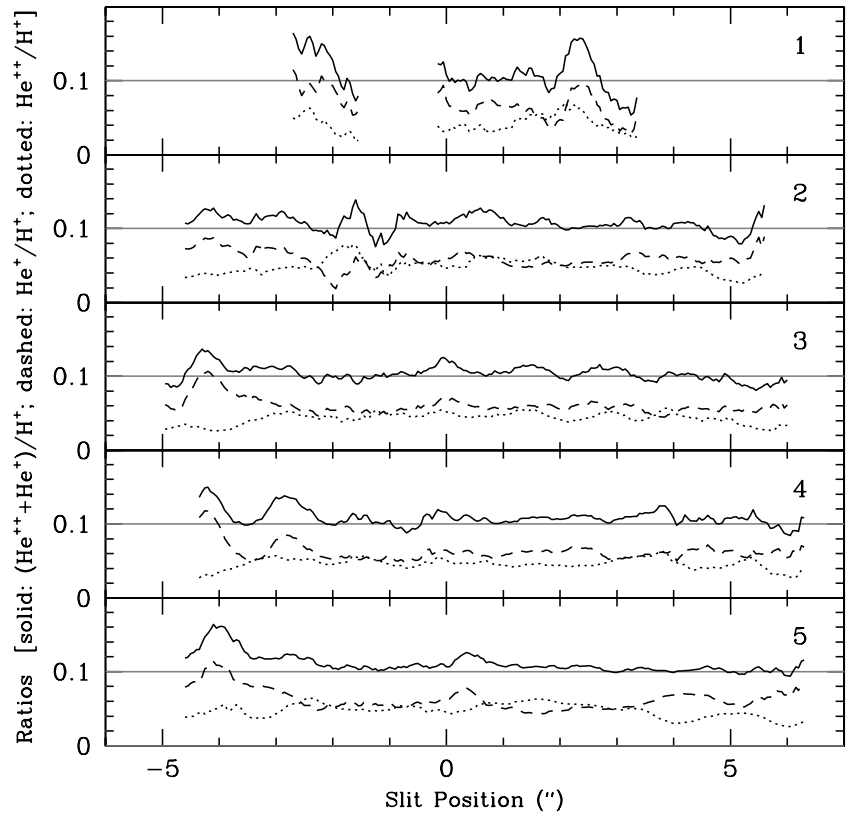

FIG. 8.- Line-of-sight average ionic abundance ratios (by number density) for $\mathrm{He}^{++} / \mathrm{H}^{+}$(dotted lines), $\mathrm{He}^{+} / \mathrm{H}^{+}$(dashed lines), and $\left(\mathrm{He}^{++}+\mathrm{He}^{+}\right) / \mathrm{H}^{+}$(solid lines) derived from the line intensities in Fig. 7. The horizontal line at 0.10 shows the nebular average value for $\left(\mathrm{He}^{++}+\mathrm{He}^{+}\right) / \mathrm{H}^{+}$derived by Zhang et al. (2005).

$2-5$, some $10 \%-15 \%$ of the pixels shown in Figure 8 have a total helium ratio $\left(\mathrm{He}^{++}+\mathrm{He}^{+}\right) / \mathrm{H}^{+}$more than $20 \%$ higher than the nebular average of 0.10 . These positions are mostly at offsets more negative than $-3.5^{\prime \prime}$ in slits 3,4 , and 5 , and show increased $\mathrm{He}_{\mathrm{I}}$ $\lambda 5876$ emission relative to positions at more positive offsets (see Fig. 7). The largest discrepancy occurs in slit 5 at $-4.0^{\prime \prime}$, where $\left(\mathrm{He}^{++}+\mathrm{He}^{+}\right) / \mathrm{H}^{+}$reaches 0.16 , or $60 \%$ higher than the nebular average value. From the published instrument specifications, it is unlikely that this discrepancy is due to flux calibration errors along the G750L slit, but we cannot rule out this possibility. In subsequent discussion, we will therefore regard with caution line fluxes from the G750L grating over these limited portions of the slits.

\subsection{Density Indicators}

We attempted to use the [S II] doublet ratio of $\lambda 6716$ to $\lambda 6731$ intensities to derive a line-of-sight average electron density $n_{e}$ at each pixel for the five slit positions. The density was calculated using the IRAF task temden (Shaw \& Dufour 1995), which is based on the formalism of de Robertis et al. (1987). The measured intensities were boxcar smoothed by 11 pixels $\left(0.55^{\prime \prime}\right)$ before subtracting the continuum and computing the line ratios. The resulting dereddened and continuum-corrected line intensity profiles and ratio are shown in Figure 9. We assumed a temperature of $13,000 \mathrm{~K}$ but the densities derived from the [S II] doublet are quite insensitive to temperature, especially toward the high-density limit for this line ratio $\left(\log n_{e}>4\right)$. The $[\mathrm{S}$ II] doublet ratios, $I(\lambda 6716) / I(\lambda 6731)$, were $\leq 0.5$ at essentially all pixels with sufficiently small uncertainties $(<10 \%$ in the ratio) to be useful as density indicators. A line ratio of 0.5 corresponds to $\log n_{e}=4.1$; a $10 \%$ increase in the ratio corresponds to a factor of 2 decrease in $n_{e}$, while a $10 \%$ decrease in the ratio gives only a lower limit to $n_{e}$. The $[\mathrm{S}$ II] lines are so close in wavelength that errors in the doublet ratio due to the reddening correction and flux calibration should be negligible, but the derived densities are very sensitive for ratios $<0.5$ so we regard the values of $n_{e}$ inferred from [S II] only as lower limits with $n_{e}>10^{4} \mathrm{~cm}^{-3}$. Other observations of the $\left[\mathrm{S}_{\mathrm{II}}\right]$ doublet ratio, made with large apertures, also found

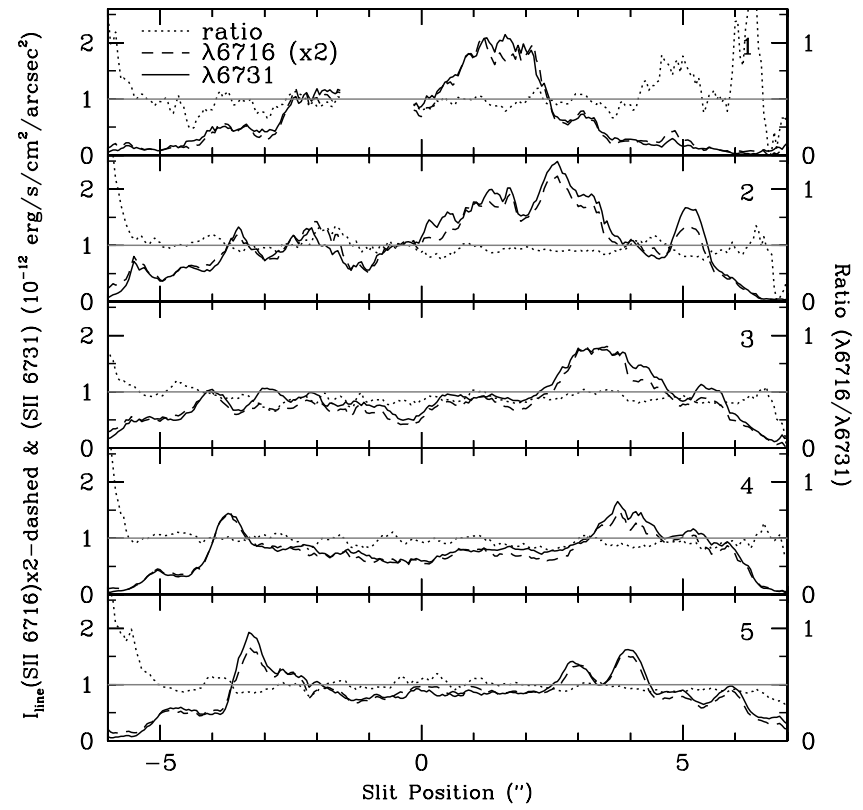

FIG. 9.-Dereddened and continuum-corrected intensity profiles for [S II] $\lambda 6716$ (dashed lines) and $\lambda 6731$ (solid lines), and corresponding line ratio (dotted lines, right axis). Data were boxcar smoothed by 11 pixels $\left(0.55^{\prime \prime}\right)$ before applying corrections.

essentially saturated values giving lower limits to the average density. Keyes et al. (1990) found a [S II] ratio of 0.435 over the brightest region of the nebula and inferred a density $n_{e} \sim 30,000 \mathrm{~cm}^{-3}$ from this diagnostic. Zhang et al. (2005) found a ratio of 0.444 and quote only a lower limit of $10^{5} \mathrm{~cm}^{-3}$ for spectra integrated over the whole nebula. They suggested that the [S II] doublet is not very suitable for density determination in NGC 7027. (In contrast, however, Bains et al. [2003] found from their velocityresolved long-slit spectra that the region they identified as the interior of the ionized shell had $n_{e} \sim 6 \times 10^{3} \mathrm{~cm}^{-3}$ and found the [S II] doublet was not saturated everywhere.)

We therefore used a second density indicator, the [Ar IV] $\lambda 4711 /$ $\lambda 4740$ doublet ratio, which saturates at densities of $\sim 10^{6} \mathrm{~cm}^{-3}$. These [Ar IV] lines are detected in our G430L grating exposures, albeit with modest signal-to-noise ratios, but by applying some pixel averaging we did obtain useful densities at many pixels.

With our spectral extraction aperture width of $20 \AA$, the [Ar IV] $\lambda 4711$ line is blended with a He I recombination line at $4713 \AA$, and with two [Ne IV] lines at 4714 and $4716 \AA$ (Zhang et al. 2005). These lines contribute about $45 \%$ of the total flux within our spectral aperture for [Ar IV] $\lambda 4711$. (The $\lambda 4740$ line has no significant contamination at this aperture width.) To correct for the contribution of these $\mathrm{He}_{\mathrm{I}}$ and $\left[\mathrm{Ne} \mathrm{IV}\right.$ ] lines, we estimate the $\mathrm{He}_{\mathrm{I}} \lambda 4713$ line intensity at each pixel by scaling our dereddened He I $\lambda 5876$ intensity profiles by the ratio of $I\left(\mathrm{He}_{\mathrm{I}} \lambda 4713\right) / I(\mathrm{He}$ I 25876$)$ from the nebular-averaged spectrum of Zhang et al. (2005). Similarly, we estimate the sum of the [Ne IV] $\lambda \lambda 4714,4716$ lines at each pixel by scaling our dereddened $I(\mathrm{He}$ II $\lambda 4686)$ with line ratios of Zhang et al. (2005). Since $\mathrm{Ne}^{++}$and $\mathrm{He}^{+}$are ions with roughly comparable ionization potentials (63.5 and $54.4 \mathrm{eV}$, respectively), the [Ne IV] line intensities should track He II $\lambda 4686$ reasonably well across the nebula, and better than [Ne IV] tracks $\mathrm{H} \alpha$. These estimates of the He I and [ $\mathrm{Ne}$ IV] contributions were subtracted from the dereddened [Ar IV] $\lambda 4711$ profile.

We estimated the continuum at $\lambda \lambda 4711,4740$ by scaling to the dereddened spatial intensity profiles of: (1) $\mathrm{H} \alpha$ for free-free (f-f) and bound-free ( $\mathrm{b}-\mathrm{f})$ emission of $\left(\mathrm{H}^{+}, e^{-}\right)$as well as the twophoton continuum; (2) He II $\lambda 4686$ spatial profiles for $\mathrm{f}-\mathrm{f}$ and $\mathrm{b}-\mathrm{f}$ 


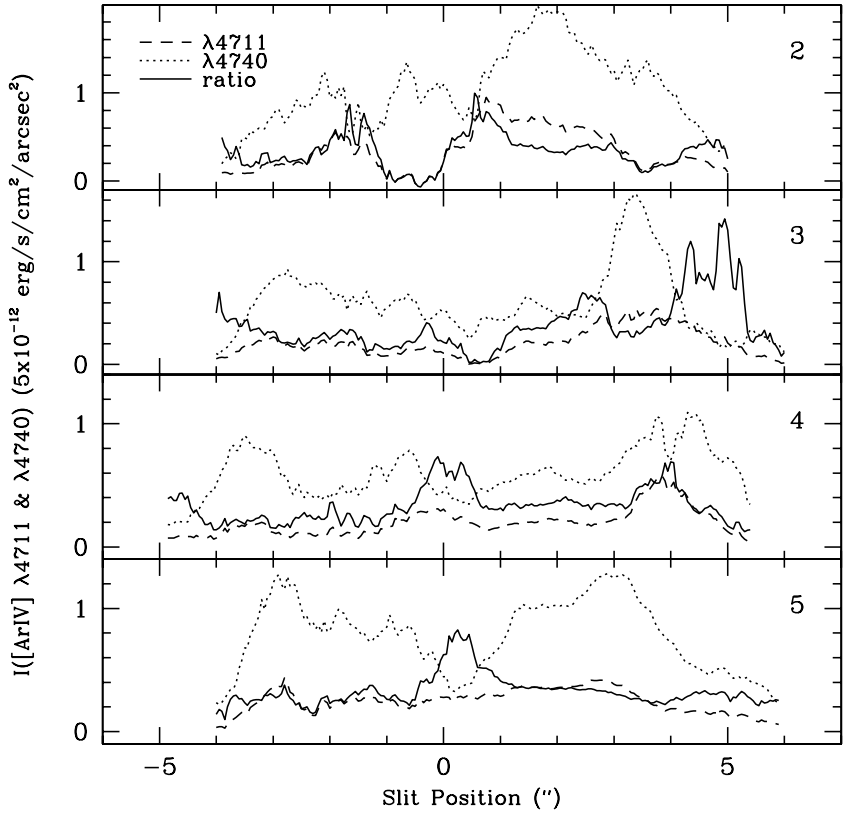

FIG. 10.-Dereddened and continuum-corrected intensity profiles for [Ar IV] $\lambda 4711$ (dashed lines) and $\lambda 4740$ (dotted lines), and corresponding line ratio (solid lines). Data were boxcar smoothed by 21 pixels $\left(1.05^{\prime \prime}\right)$ before applying corrections.

processes of $\left(\mathrm{He}^{++}, \mathrm{e}^{-}\right)$; and (3) He I $\lambda 5876$ spatial profiles for $\mathrm{f}-\mathrm{f}$ and $b$-f processes of $\left(\mathrm{He}^{+}, \mathrm{e}^{-}\right)$. Scaling factors were derived from the tables of line and continuum emissivities in Osterbrock \& Ferland (2006), by interpolating to an assumed $T_{e}=13,000 \mathrm{~K}$ and adopting the nebular-averaged ionic abundances from Zhang et al. (2005). The total continuum summed over these three processes was subtracted at each pixel from both $I(\mathrm{Ar}$ IV 24711$)$ and I(Ar IV 24740). The corrected [Ar IV] $\lambda 4711$ intensity was ratioed with [Ar IV] $\lambda 4740$. The corrected line intensity profiles and resulting line ratios are shown in Figure 10. Most of the slit positions have line ratios around $0.2-0.4$, consistent with the global average ratio of Zhang et al. (2005), who found $I(\lambda 4711) / I(\lambda 4740)=$ 0.273 . There are isolated regions, however, which show line ratios significantly larger than the average, notably slit 3 near $+2.7^{\prime \prime}$ and $\left(4^{\prime \prime}\right.$ to $\left.+5.3^{\prime \prime}\right)$; slit 4 near $0^{\prime \prime}$ and $\left(+3.5^{\prime \prime}\right.$ to $\left.4^{\prime \prime}\right)$; and slit 5 from $0^{\prime \prime}$ to $+0.5^{\prime \prime}$.

Density profiles were derived from this ratio using the IRAF temden task, as for the [S II] doublet. Figure 11 shows the resulting line-of-sight average densities for slits 2-5 from the [Ar IV] doublet. Useful mean density values were obtained for a fraction of the measured pixels on slits $2-5$. The weak [Ar IV] lines and the additional correction for the $\mathrm{He}$ I and [ $\mathrm{Ne}$ IV] lines result in relatively large uncertainties for the densities, except in the brighter parts of the nebula sampled by the slits. The densities are distributed about the nebular average $\left(\log n_{e}=4.77\right.$; Zhang et al. 2005). Where the line ratio is small and the lines are weak, the densities are not well determined, giving $\log n_{e} \sim 5$. Isolated sections show densities over regions $\sim 1^{\prime \prime}$ in size (comparable to the smoothing length) with $\log n_{e} \sim 4$, corresponding to the slit locations with larger than average line ratios noted above.

\subsection{Temperature Indicators}

We determined line-of-sight mean electron temperatures, $T_{e}$, from the ratios of intensities of [O III] $[I(4959 \AA)+I(5007 \AA)] /$ $I(4363 \AA)$, assuming a constant density of $n_{e}=60,000 \mathrm{~cm}^{-3}$, after boxcar smoothing the spatial profiles by 11 pixels $\left(0.55^{\prime \prime}\right)$. This assumed density is consistent with the nebular average determined by Zhang et al. (2005) from several diagnostics, including

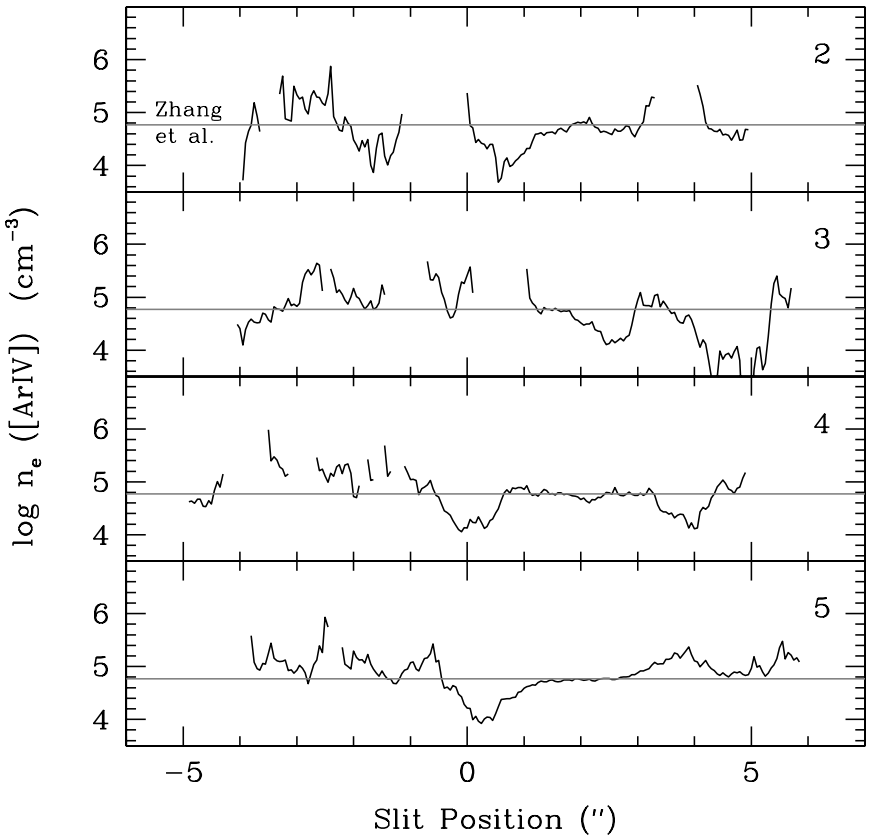

FIG. 11.-Electron densities derived from [Ar IV] $\lambda 4711 / \lambda 4740$ line ratios in Fig. 10. Values are blanked where the line ratio is saturated at 0.18 , indicating $\log n_{e}>6$. Thin horizontal line: Nebular average density obtained by Zhang et al. (2005).

[Ar IV]. The derived temperatures are relatively insensitive to the assumed $n_{e}$, provided it is below the critical density of $10^{5} \mathrm{~cm}^{-3}$ (Osterbrock 1989). For example, reducing $n_{e}$ by a factor of 2 results in a $\sim 5 \%$ increase in $T_{e}$. Results for slit positions $2-5$ are shown in Figure 12. Temperatures shown are restricted to pixels where the dereddened $I(\mathrm{H} \alpha)>2 \times 10^{-11} \mathrm{erg} \mathrm{s}^{-1} \mathrm{~cm}^{-2} \operatorname{arcsec}^{-2}$.

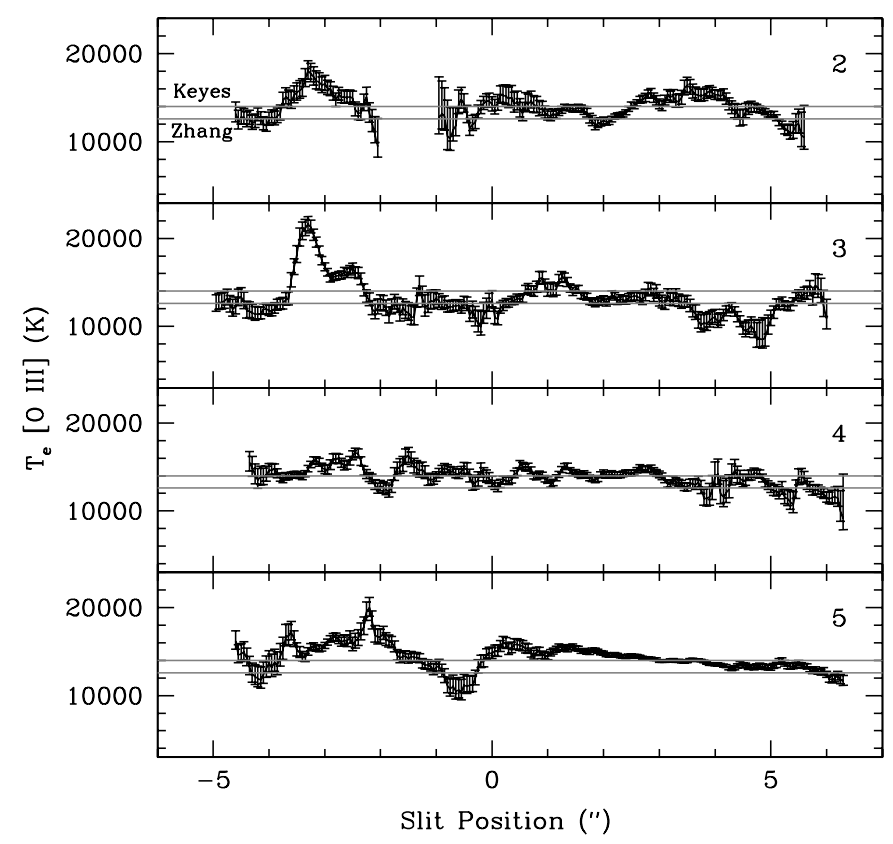

FIG. 12.-Electron temperatures derived from [O $\mathrm{III}](\lambda 4959+\lambda 5007)$ $\lambda 4363$, as a function of slit position for slit locations $2-5$ shown in Fig. 1. Density was taken as fixed at $60,000 \mathrm{~cm}^{-3}$. Data were boxcar averaged over 11 pixels $\left(0.55^{\prime \prime}\right)$ along the slit before calculating temperature. Error bars are $1 \sigma$. Horizontal lines show the average values derived by Keyes et al. (1990) for the optically brightest part of the nebula, and by Zhang et al. (2005) for the whole nebula. The overall mean of the plotted points is $13,850 \mathrm{~K}$, i.e., very close to the Keyes et al. value of $14,000 \mathrm{~K}$. 


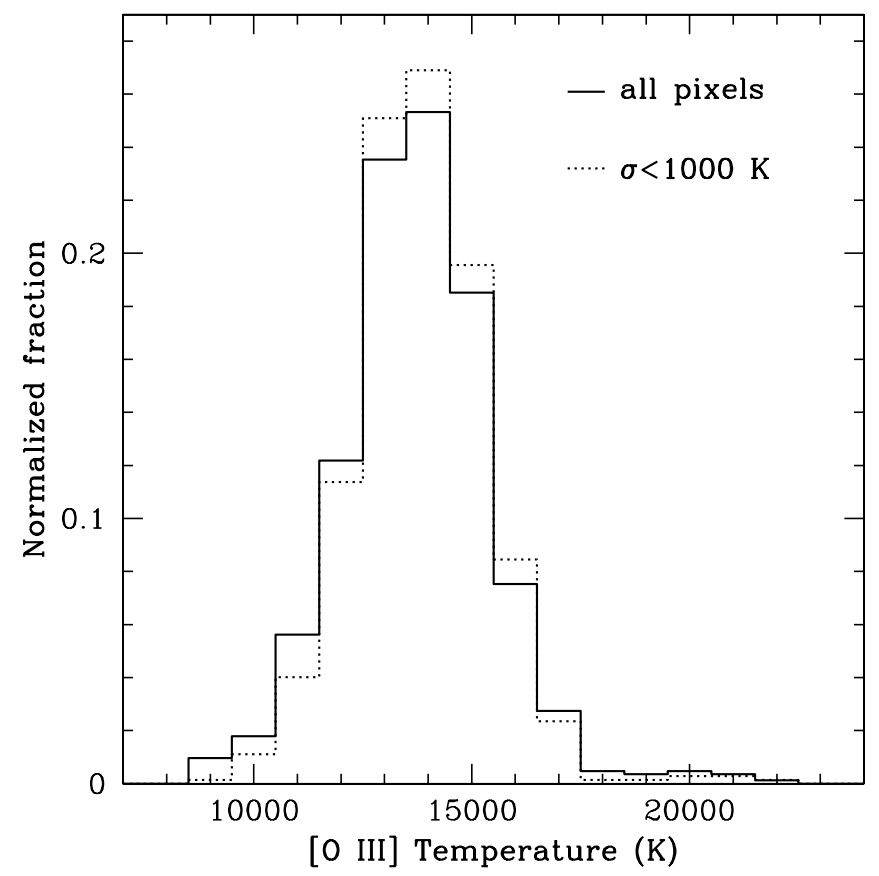

FIG. 13. - Histograms of [O III]-derived temperatures over slits $2-5$, as shown in Fig. 12. Dotted line: Pixels where $1 \sigma$ uncertainties for the derived temperatures were not larger than the bin width of $1000 \mathrm{~K}$. Solid line: All pixels in Fig. 12 are included.

This intensity cutoff restricts the plotted points to pixels with formal errors in $T_{e}$ less than $\sim 2000 \mathrm{~K}$. We also omit $\sim 1^{\prime \prime}$ along slit 2 where errors in $T_{e}$ exceed $3000 \mathrm{~K}$.

The $[\mathrm{O}$ III] temperatures are generally much better determined than are the densities, since the $[\mathrm{O}$ III] lines are bright compared to the density indicators. The spatial profiles are relatively flat, with average values near $14,000 \mathrm{~K}$ in each slit. For comparison, the mean values of $T_{e}$ obtained from the [O III] lines by Keyes et al. (1990) for the brightest part of the nebula, and by Zhang et al. (2005) for the entire nebula, are shown as horizontal lines in Figure 12. There are deviations from a constant $T_{e}$ by $>1 \sigma$ for some ranges of positions along the slits, suggesting that spatial variations in $T_{e}$ may be present in the nebula. Locations which show excursions from the mean that appear to be statistically significant $(>2 \sigma)$ are in slit 3 at $-3.4^{\prime \prime}$ and slit 5 at $-2.2^{\prime \prime}$.

The distribution of $T_{e}$ values from the [O III] lines is shown in Figure 13 as a histogram. Two samples (not independent) are plotted: all pixels for which the formal uncertainty in $T_{e}$ is $\sigma<1000 \mathrm{~K}$, and all pixels in Figure 12 regardless of formal uncertainty. The more restricted sample (dotted line) has a mean $\left\langle T_{e}\right\rangle=13,850 \mathrm{~K}$ with a standard deviation $\sigma\left(T_{e}\right)=1520 \mathrm{~K}$, slightly larger than the selection criterion on the formal error. Taking the difference in quadrature between the observed standard deviation and the sample selection limit would imply an intrinsic spread in $T_{e}$ for those pixels of about $\sigma_{\text {intr }}\left(T_{e}\right)=1140 \mathrm{~K}$. The unrestricted sample (solid line) has $\left\langle T_{e}\right\rangle 13,800 \mathrm{~K}$ and $\sigma\left(T_{e}\right)=1710 \mathrm{~K}$, consistent with the larger spread in uncertainties of individual $T_{e}$ values. There appears to be a non-Gaussian wing extending to higher $T_{e}$ values in both samples, but this wing contains a small fraction of the unrestricted distribution; $2 \%$ of the included points have $T_{e}>18,000 \mathrm{~K}$.

The [S III] lines at 9069 and $9531 \AA$ were detected in the long wavelength end of the G750L grating spectra. Together with the [S III] $6312 \AA$ line extracted from the G750M grating spectra, these form another diagnostic for nebular temperature. We calculated $T_{e}$ from the [S III] lines as for [O $\left.\mathrm{III}\right]$, but found that the results had a mean $\left\langle T_{e}\right\rangle$ that was $1500 \mathrm{~K}$ higher than for [O III] for the same pixels as in Figure 12, and the dispersion of values for an unrestricted sample was $40 \%$ higher than for the [O III] values. Moreover, there was no evident correlation between the variations in the [O III]- and [S III]-derived $T_{e}$ values. We conclude that for the present data set the [O III] lines must be preferred over [S III] as diagnostics of $T_{e}$. The larger scatter in the [S III] temperatures may result from several effects related to the larger fractional wavelength differences in the [S III] lines $(\sim 40 \%)$ compared to the [O III] lines ( 14\%): (1) residual errors in the reddening correction as a function of slit positions; (2) residual flux calibration errors as a function of slit position; and (3) differential errors in calibration of the $\mathrm{G} 750 \mathrm{~L}$ and $\mathrm{G} 750 \mathrm{M}$ grating spectra. (The $[\mathrm{S}$ III] $\lambda 6312$ line, although present in the G750L spectrum, was blended with [O I] $\lambda 6300$, so it was necessary to extract it from the higher resolution G750M grating. The $[\mathrm{O}$ III] lines, in contrast, were all from the same grating.)

\section{DISCUSSION}

\subsection{Spatial Variations in Temperature and Density}

One of the objectives of this study was to look for evidence of spatial variations of $T_{e}$ and $n_{e}$ within the ionized gas of NGC 7027. The plots of $T_{e}[\mathrm{O}$ III] versus slit position (Fig. 12) show only a few positions at which $T_{e}$ differs from the mean by more than twice the formal measurement uncertainties, suggesting that deviations from an isothermal nebula are limited in extent. From the histograms in Figure 13, we found (see $\$ 3.3$ ) that the intrinsic rms scatter in the derived temperatures which have formal uncertainties $<1000 \mathrm{~K}$ due to photon noise statistics was $\sim 1140 \mathrm{~K}$ for $T_{e}[\mathrm{O}$ III], with a mean value of $13,850 \mathrm{~K}$. This excess in the rms amounts to only about $8 \%$ of the mean $T_{e}$, however, which may be attributable principally to a combination of residual calibration errors and errors in the reddening corrections. The histogram bins in Figure 13 with $T_{e} \geq 18,000 \mathrm{~K}$ correspond to one or two "hot spots" in slits 3 and 5 , but these comprise only about $2 \%$ of the total pixels included.

We can also consider the observed temperature variations in terms of Peimbert's $t^{2}$ formalism, following Liu's (1998) suggestion that a "surface temperature variance" parameter, $t_{s}^{2}$, should be a lower limit to the three-dimensional $t^{2}$ defined by Peimbert (1967). We calculated

$$
t_{s}^{2} \equiv \frac{\sum\left[\left(T_{e}, i[\mathrm{O} \mathrm{III}]-T_{o}\right)^{2} I_{i}(\mathrm{H} \alpha)\right]}{T_{o}^{2} \sum I_{i}(\mathrm{H} \alpha)},
$$

i.e., the variance of the observationally derived $T_{e}[\mathrm{O}$ III $]$ weighted by the $\mathrm{H} \alpha$ intensity, where the summations are over pixels, $i$. Here, $T_{o}$ is the mean value of $T_{e}[\mathrm{O}$ III] weighted by $I(\mathrm{H} \alpha)$. ( We used $I(\mathrm{H} \alpha)$ rather than $I(\mathrm{H} \beta)$ because of smaller reddening and the superior signal-to-noise ratio.) We derived $t_{s}^{2}=0.011$ averaging over all pixels for slits $2-5$ shown in Figure 12. Liu (1998) argues that this observable quantity, $t_{s}^{2}$, is a lower limit to $t^{2}$, which Peimbert (1967) defined by weighting $T_{e}$ by $n_{e}^{2}$ at each point in the nebula. In a theoretical study, Capetti (2006) showed that for spherical models, the $t_{s}^{2}$ for $T[\mathrm{O}$ III] was only $\sim 25 \%$ of the true three-dimensional $t^{2}$ parameter. Without a priori knowledge of the actual temperature and density distributions, it is impossible to say how $t_{s}^{2}$ and $t^{2}$ are related, except that our observed $t_{s}^{2}$ is a firm lower limit to $t^{2}$, but by an unknown factor.

The existing abundance data for NGC 7027 do not indicate that large temperature variations need to be invoked. Bernard Salas et al. (2001), using data from Keyes et al. (1990), found that $\mathrm{C}^{++}$, $\mathrm{N}^{++}$, and $\mathrm{O}^{++}$abundance discrepancy factors (ADFs) were between 
1.1 and 2.4 from recombination lines compared to collisionally excited lines, but they dismissed the differences as insignificant. From their sensitive high-resolution nebular-averaged spectrum, Zhang et al. (2005) found that $\mathrm{C}^{++}, \mathrm{N}^{++}, \mathrm{O}^{++}$, and $\mathrm{Ne}^{++}$showed ADFs between 1.2 and 1.7. These relatively small ADFs are consistent with trends versus nebular age, where young PNs such as NGC 7027 yield abundance values which are consistent between recombination and collisionally excited lines. Spatial variations in $T_{e}$ are not necessarily excluded by these consistent abundance determinations, but the relatively small ADFs do not imply that such variations must be present.

The density profiles derived from the [Ar IV] diagnostic (Figs. 10 and 11) do show evidence for variations in the line-of-sight averaged densities within small spatial scales. Changes by factors of $\sim 5-7$ within $\sim 1^{\prime \prime}$ along a slit are evident at several locations. Our data do not appear to be consistent with a uniform density nebula, such as the Masson (1989) model based on radio continuum data assumed. Other observations at radio and IR wavelengths, i.e., with little or no effect of extinction, also indicate that the density of the ionized gas is not uniform. The velocity resolved $\mathrm{Br} \gamma$ images of Cox et al. (2002) show that numerous small-scale highdensity clumps must be present in a uniform shell-like structure. The subarcsecond resolution radio continuum images by Bryce et al. (1997) and Bains et al. (2003) also show small-scale structure in the radio brightness temperature, which these authors argue implies the existence of high-density clumps.

Density variations within the ionized gas may reflect density structure that was present within the neutral stellar wind material ejected while the central star was on the AGB. Both CO and scattered light observations of AGB and post-AGB objects reveal considerable structure in the ejecta from late-type stars. The shell structures found around, e.g., AFGL 2688 (Sahai et al. 1998) and IRC +10216 (Mauron \& Huggins 1999) indicate that the outflow has quasi-periodic oscillations, with density contrast corresponding to factors of 100-1000 (Schöier et al. 2005; Decin et al. 2006). These mass-loss modulations on a timescale of a few hundred years may result from a complex feedback between hydrodynamics, gas/dust drift, and gas-solid chemistry and so might be a common aspect of AGB winds (Simis et al. 2001). Alternatively, variations in the ionized gas of NGC 7027 could result from the interaction of the fast, hot, tenuous wind with the previously ejected slow, cold, dense AGB wind. This situation would be expected to produce Rayleigh-Taylor instabilities at the interface, which could generate clumps of high density within the more diffuse shell. The X-ray data (Kastner et al. 2001; Maness et al. 2003) attest to the possible importance of this interaction in NGC 7027.

\subsection{Ionization Inhomogeneities}

There is evidence in our data for spatial variations in the ionization state of the gas, which is hardly surprising given the complex nature of this object. The ratio of $\mathrm{He}^{++} / \mathrm{H}^{+}$shown in Figure 8 changes by factors of $\sim 1.5-2$ from center to the outer parts of the nebula. For example, in slit 5 from $+1.5^{\prime \prime}$ to $+6.0^{\prime \prime}$ the line-of-sight averaged fraction of $\mathrm{He}^{++} / \mathrm{H}^{+}$falls from 0.06 to 0.03 . For slits 2 through 5, the $\mathrm{He}^{++}$fraction lies in the range $0.04-0.06$ for slit positions between $-3^{\prime \prime}$ and $+4^{\prime \prime}$, and drops to $\sim 0.02-0.03$ at the largest offset positions $\left(-5^{\prime \prime}\right.$ and $\left.+6^{\prime \prime}\right)$.

The distribution of intensities of the low-ionization indicator [O I ] $\lambda 6300$ also shows evidence for variations in the ionization state with position. Figure 14 plots $I\left(\left[\mathrm{O}_{\mathrm{I}}\right]\right.$ 26300) compared with $I(\mathrm{H} \alpha)$. Both the He II (Fig. 7) and [O I] (Fig. 14) lines are coextensive with $\mathrm{H} \alpha$, but with small-scale variations in relative intensity. Most of the distinct $\mathrm{H} \alpha$ peaks have counterparts in both

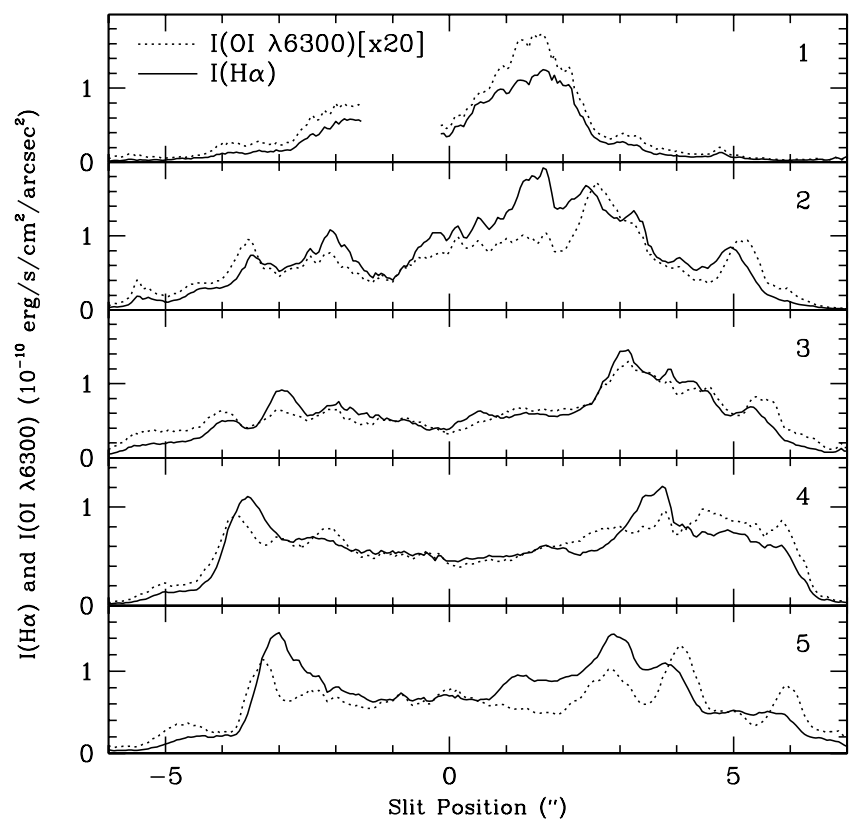

FIG. 14.- Comparison of 11 pixel $\left(0.55^{\prime \prime}\right)$ boxcar-smoothed, dereddened intensity profiles for each slit (numbers at upper right) showing $\mathrm{H} \alpha$ (solid line), and [O I] $\lambda 6300$ multiplied by a factor of 20 (dotted line).

He II and [O I]. In several positions we see a pattern of displacement of the emission peaks in the sense that He II tends to lie either coincident with or interior to the peak of $\mathrm{H} \alpha$, i.e., closer to the central star as projected on the plane of the sky. In contrast, $\left[\mathrm{O}_{\mathrm{I}}\right] \lambda 6300$ features tend to lie to the exterior of the $\mathrm{H} \alpha$ peaks, i.e., farther from the central star in projected distance on the plane of the sky. Examples include: slit 2, $\mathrm{H} \alpha$ peaks at $+4.9^{\prime \prime}$; slit 3, at $+5.3^{\prime \prime}$; slit 4, at $-3.6^{\prime \prime}$; and slit 5 , at $-3.1^{\prime \prime},+3.8^{\prime \prime}$, and $+5.8^{\prime \prime}$. The spatial displacements of high and low ionization energy tracers in these features suggest that there are ionization gradients within the gas traced in $\mathrm{H} \alpha$, with the highest ionization on the side toward the central star. One interpretation could be that the peaks in [O I] $\lambda 6300$ represent "ionization shadows" and the peaks in He II $\lambda 4686$ trace the inward side of clumps directly exposed to the $200,000 \mathrm{~K}$ central star radiation field.

Shock excitation may also play a role, however. The intensity of [O I] $\lambda 6300$ in NGC 7027 is typically $\sim 15 \%$ of $\mathrm{H} \beta$ [see Fig. 14, with $I(\mathrm{H} \alpha) / I(\mathrm{H} \beta) \approx 3]$, a value at the upper end of the range for planetary nebulae in the compilation of Phillips \& Guzman (1998) for objects of comparable physical dimensions (and therefore, presumably, evolutionary state). Phillips \& Guzman argue that strong [O I] $\lambda 6300$ emission must arise from dense gas in the precursor AGB star wind being shocked by a fast wind from the central star. NGC 7027 has formed and is evolving within a massive neutral molecular envelope ejected by the star (Bieging et al. 1991; Jaminet et al. 1991), so the ionization structure is probably developing under the combined effects of stellar radiation and shocks from a fast stellar wind.

A physically realistic model for the ionization structure of NGC 7027 presents a formidable challenge. The central star has an effective temperature of $200,000 \mathrm{~K}$, but the extended X-ray emission (Kastner et al. 2001) should be considered as an additional diffuse source of ionizing photons. The presence of collimated fast outflows or jets seems well established observationally (Latter et al. 2000; Cox et al. 2002), and a fast isotropic $\left(\sim 1000 \mathrm{~km} \mathrm{~s}^{-1}\right)$ wind from the central star may be present as well. The interaction of such jets or stellar wind with the slowly expanding stellar 
envelope would be expected to produce collisionally ionized gas by shocks. Optical and near-IR images in both continuum and emission lines show that the ionized shell of the PN is clumpy, not smooth. Our results for the line-of-sight average density distribution along each slit position also suggest that the ionized shell contains a range of densities, which vary over small spatial scales (comparable to the binned resolution of $1.05^{\prime \prime}$ or $\sim 10^{16} \mathrm{~cm}$ ). Such inhomogeneities in the presence of a dynamically evolving nebula could be expected to produce spatial variations in the ionization state of the gas, as suggested by the spatial distribution of $\mathrm{He}$ II $\lambda 4686$ and [O I] $\lambda 6300$ relative to $\mathrm{H} \alpha$.

\section{SUMMARY}

We observed the young planetary nebula NGC 7027 with the HST Imaging Spectrograph (STIS) in long-slit mode at five slit positions oriented parallel to the major axis of the nebula and spaced at regular intervals from the edge to the brightest part of the ionized gas. The slit width was $0.1^{\prime \prime}$ with spatial sampling of $0.05^{\prime \prime}$. Three grating settings covered the wavelength range from 3000 to $\sim 10,000 \AA$ in low-resolution mode $(\Delta \lambda \sim 5-10 \AA)$ and from 6300 to $6850 \AA$ at medium resolution $(\Delta \lambda \sim 1 \AA)$.

We used dereddened line ratios to determine line-of-sight average values for (1) $\mathrm{He}^{++}$and $\mathrm{He}^{+}$ionization fraction from $\mathrm{He}$ II $\lambda 4686$ and $\mathrm{He}$ I $\lambda 5876$; (2) electron density from both [ $\left.\mathrm{S}_{\mathrm{II}}\right](\lambda 6716 /$ $\lambda 6731)$ and $[\mathrm{Ar} \mathrm{IV}](\lambda 4711 / \lambda 4740)$; and (3) temperature from [O III] $(\lambda 4959+\lambda 5007) / \lambda 4363$. Generally we used spatial smoothing along the slit to reduce the noise, so typical resolutions along each slit were $0.55^{\prime \prime}$.

The $[\mathrm{S} \mathrm{II}]$ doublet is saturated where it can be measured sufficiently well, so yields lower limits of $n_{e}>10^{4} \mathrm{~cm}^{-3}$. The [Ar IV] doublet ratio, which saturates at $\sim 10^{6} \mathrm{~cm}^{-3}$, gives well-determined (factor of 2 uncertainty) line-of-sight averaged densities ranging from 10,000 to $\sim 100,000 \mathrm{~cm}^{-3}$. Higher values are indicated along many sight lines, but have large uncertainties because the doublet ratio approaches saturation. The $[\mathrm{Ar}$ IV] line intensities show variations which imply mean density variations by factors of $\sim 5$ down to the smallest angular scales set by the spatial smoothing $\left(\sim 1^{\prime \prime}\right)$.

The line-of-sight averaged electron temperatures at positions along the slits using the $[\mathrm{O} \mathrm{III}](\lambda 4959+\lambda 5007) / \lambda 4353$ diagnostic are generally consistent with an overall $\left\langle T_{e}\right\rangle=13,000 \mathrm{~K}$. At one or two locations $T_{e}$ exceeds $18,000 \mathrm{~K}$ but these comprise only $\sim 2 \%$ of all pixels with formal errors $\sigma\left(T_{e}\right)<2000 \mathrm{~K}$. A histogram of $T_{e}([\mathrm{O} \mathrm{III}])$ values with $1 \sigma$ uncertainties $<1000 \mathrm{~K}$ has a mean of $13,850 \mathrm{~K}$ and a standard deviation of $1520 \mathrm{~K}$. The resid- ual scatter in excess of the formal error due to photon noise is $\sim 8 \%$ of the mean $T_{e}$, and may be attributed largely to residual errors in calibration and reddening corrections.

Our spatial sampling of the nebula is limited to the five slit positions, so it is difficult to make strong conclusions about correlations of the temperature and density variations with the extinction features which are so evident in the HST optical images (e.g., Fig. 1). However, there is no indication that the variations of temperature and density along the slits are correlated with variations in extinction.

The ionization structure of the nebula varies on both large and small scales. The average $\mathrm{He}^{++} / \mathrm{H}^{+}$ratio is in the range $0.04-0.06$ for positions within $4^{\prime \prime}$ of the central star, and drops to $0.02-0.03$ at slit positions $\geq 5^{\prime \prime}$ from the center. Comparison of [O I ] $\lambda 6300$, $\mathrm{H} \alpha$, and $\mathrm{He}$ II $\lambda 4686$ intensity profiles along each slit provides further evidence for small-scale ionization structures. Several distinct $\mathrm{H} \alpha$ emission peaks appear to have ionization gradients, with the highest ionization on the side toward the central star and lowest ionization on the far side.

We suggest that the observed variations in the ionization structure (and to the extent they are present, in electron temperature) can be interpreted in terms of density-driven variations in a nebula photoionized by the central star, possibly assisted by the diffuse $\mathrm{X}$-ray emission from the shocked stellar wind. Alternatively, shocks driven into this inhomogeneous environment by the observed stellar jets may produce temperature fluctuations as well. Detailed modeling is required to fully understand the physical conditions, structure, and evolution of such a dynamically evolving nebula and is imperative to derive accurate elemental abundances. A more complete picture of these structural details awaits further highsensitivity observations with both high spatial and spectral resolution, such as will be feasible with adaptive optics and imaging spectrographs on ground-based telescopes at near-IR and optical wavelengths.

We thank Indra Bains for providing her emission measure map in digital form, and Don Garnett for helpful discussions. Support for proposal number GO-08285 was provided by NASA through a grant from the Space Telescope Science Institute, which is operated by the Association of Universities for Research in Astronomy, Incorporated, under NASA contract NAS5-26555. This work was supported in part by National Science Foundation grant AST0307687 to The University of Arizona.
Atherton, P. D., Hicks, T. R., Reay, N. K., Robinson, G. J., Worswick, S. P., \& Phillips, J. P. 1979, ApJ, 232, 786

Bains, I., Bryce, M., Mellema, G., Redman, M. P., \& Thomasson, P. 2003, MNRAS, 340, 381

Benjamin, R. A., Skillman, E. D., \& Smits, D. P. 1999, ApJ, 514, 307

Bernard Salas, J., Pottasch, S. R., Beintema, D. A., \& Wesselius, P. R. 2001, A\&A, 367, 949

Bieging, J. H., Wilner, D., \& Thronson, H. A. 1991, ApJ, 379, 271

Blöcker, T. 1995, A\&A, 299, 755

Bryce, M., Pedlar, A., Muxlow, T., Thomasson, P., \& Mellema, G. 1997, MNRAS, 284, 815

Capetti, M. V. F. 2006, A\&A, 453, 943

Ciardullo, R., Bond, H. E., Sipior, M. S., Fullton, L. K., Zhang, C.-Y., \& Schaefer, K. G. 1999, AJ, 118, 488

Cox, P., Huggins, P. J., Maillard, J.-P., Habart, E., Morisset, C., Bachiller, R., \& Forveille, T. 2002, A\&A, 384, 603

Decin, L., Hony, S., de Koter, A., Justtanont, K., Tielens, A. G. G. M., \& Waters, L. B. F. M. 2006, A\&A, 456, 549

de Robertis, M. M., Dufour, R. J., \& Hunt, R. W. 1987, J. R. Astron. Soc. Canada, 81, 195

Garnett, D. R., \& Dinerstein, H. L. 2001, ApJ, 558, 145

\section{EFERENCES}

Graham, J. R., Serabyn, E., Herbst, T. M., Matthews, K., Neugebauer, G., Soifer, B. T., Wilson, T. D., \& Beckwith, S. 1993, AJ, 105, 250

Jacoby, G. 1988, ApJ, 333, 193

Jaminet, P. A., Danchi, W. C., Sutton, E. C., Russell, A., Sandell, G., Bieging, J. H., \& Wilner, D. 1991, ApJ, 380, 461

Kastner, J. H., Vrtilek, S. D., \& Soker, N. 2001, ApJ, 550, L189

Keyes, C. D., Aller, L. H., \& Feibelman, W. A. 1990, PASP, 102, 59

Lame, N. J., \& Pogge, R. W. 1994, AJ, 108, 1860

Latter, W. B., Dayal, A., Bieging, J. H., Meakin, C., Hora, J. L., Kelly, D. M., \& Tielens, A. G. G. M. 2000, ApJ, 539, 783

Liu, X.-W. 1998, MNRAS, 295, 699

. 2003, in IAU Symp. 209, Planetary Nebulae: Their Evolution and Role in the Universe, ed. S. Kwok, M. Dopita, \& R. Sutherland (San Francisco: ASP), 339

. 2006, in IAU Symp. 234, Planetary Nebulae in our Galaxy and Beyond, ed. M. J. Barlow \& R. Mendez (Cambridge: Cambridge Univ. Press), 219 Liu, X.-W., Storey, P. J., Barlow, M. J., Danziger, I. J., Cohen, M., \& Bryce, M. 2000, MNRAS, 312, 585

Maness, H. L., Vrtilek, S. D., Kastner, J. H., \& Soker, N. 2003, ApJ, 589, 439

Masson, C. R. 1989, ApJ, 336, 294

Mathis, J. S. 1995, RevMexAA, 3, 207 
Mauron, N., \& Huggins, P. 1999, A\&A, 349, 203

Monteiro, H., Schwarz, H. E., Gruenwald, R., \& Heathcote, S. 2004, ApJ, 609, 194

Osterbrock, D. E. 1974, PASP, 86, 609

1989, Astrophysics of Gaseous Nebulae and Active Galactic Nuclei (Mill Valley: Univ. Science Books)

Osterbrock, D. E., \& Ferland, G. J. 2006, Astrophysics of Gaseous Nebulae and Active Galactic Nuclei (2nd ed.; Sausalito: Univ. Science Books)

Peimbert, M. 1967, ApJ, 150, 825

1995, in Proc. The Analysis of Emission Lines, ed. R. E. Williams \& M. Livio (Cambridge: Cambridge Univ. Press), 165

Peimbert, M., \& Peimbert, A. 2006, in IAU Symp. 234, Planetary Nebulae in Our Galaxy and Beyond, ed. M. J. Barlow \& R. H. Mendez (Cambridge: Cambridge Univ. Press), 227

Pequignot, D., Liu, X.-W., Barlow, M. J., Storey, P. J., \& Morisset, C. 2003, in IAU Symp. 209, Planetary Nebulae: Their Evolution and Role in the Universe, ed. S. Kwok, M. Dopita, \& R. Sutherland (San Francisco: ASP), 347

Phillips, J. P., \& Guzman, V. 1998, A\&AS, 130, 465

Robberto, M., Clampin, M., Ligori, S., Paresce, F., \& Staude, H. J. 1993, A\&A, 280,241

Rubin, R. H., et al. 2002, MNRAS, 334, 777
Sahai, R. 2003, in IAU Symp. 209, Planetary Nebulae: Their Evolution and Role in the Universe, ed. S. Kwok, M. Dopita, \& R. Sutherland (San Francisco: ASP), 471

Sahai, R., \& Trauger, J. T. 1998, AJ, 116, 1357

Sahai, R., et al. 1998, ApJ, 493, 301

Schöier, F., Lindqvist, M., \& Olofsson, H. 2005, A\&A, 436, 633

Seaton, M. J. 1979, MNRAS, 187, 785

Shaw, R. A., \& Dufour, R. J. 1995, PASP, 107, 896

Simis, Y. J. W., Icke, V., \& Dominik 2001, A\&A, 371, 205

Storey, P. J., \& Hummer, D. G. 1995, MNRAS, 272, 41

Torres-Peimbert, S., \& Peimbert, M. 2003, in IAU Symp. 209, Planetary Nebulae: Their Evolution and Role in the Universe, ed. S. Kwok, M. Dopita, \& R. Sutherland (San Francisco: ASP), 363

Walton, N. A., Pottasch, S. R., Reay, N. K., \& Taylor, A. R. 1988, A\&A, 200, L21

Wesson, R., \& Liu, X.-W. 2004, MNRAS, 351, 1026

Woodward, C. E., Pipher, J. L., Forrest, W. J., Moneti, A., \& Shure, M. A. 1992, ApJ, 385, 567

Zhang, Y., Liu, X.-W., Luo, S.-G., Pequignot, D., \& Barlow, M. J. 2005, A\&A, 442, 249 